\title{
On loudness enhancement of a tone burst by a preceding tone burst*
}

\author{
J. J. ZWISLOCKI and W. G. SOKOLICH \\ Institute for Sensory Research, Syracuse University, Syracuse, New York 13210
}

\begin{abstract}
The loudness level of a second tone burst in a monotic burst pair is investigated as a function of the intensity and frequency of the first burst relative to the corresponding variables of the second burst and as a function of the interburst time interval. The loudness level is measured with the help of a third, comparison burst whose frequency is the same as that of the second burst. The results, in connection with preceding results, show beyond any reasonable doubt that loudness effects in pairs of sound bursts are controlled by two perceptual processes: loudness enhancement and loudness summation. The first refers to the loudness of the second burst, the second, to the overall loudness of the burst pair. The time and frequency functions of the two processes are fundamentally different.
\end{abstract}

Several series of experiments conducted in our laboratory (Cannon \& Zwislocki, 1970; Irwin \& Zwislocki, 1971; Zwislocki \& Ketkar, 1972) have led to the conclusion that loudness effects in pairs of consecutively presented sound bursts is controlled by two distinct perceptual processes. One process, called loudness enhancement, refers to loudness changes of each burst in the pair, the other, called loudness summation, concerns the overall loudness of the pair (Zwislocki \& Ketkar, 1972). Earlier, Scharf (1969) had arrived at an analogous conclusion with respect to simultaneous heterofrequency tone bursts.

Whether listeners' responses refer to loudness enhancement or summation depends on instructions and the stimulus paradigm. In loudness matching experiments in which a third sound burst is used for loudness comparison, a loudness enhancement measure may be obtained by explicitly instructing the listeners to match the loudness of the third burst to that of the second burst and by equating the sound frequencies of the two bursts. A measure of overall loudness of a burst pair may be obtained by emphatically instructing the listeners to match the loudness of the comparison burst to the total loudness of the burst pair and by placing the sound frequency of the comparison burst at the geómetric mean of the burst-pair frequencies. When the bursts in the pair have the same frequency, a measure of their overall loudness is difficult to obtain, because the third burst has the same frequency as the second, and listeners tend to compare the loudness of the two bursts disregarding the first burst. In general, listeners tend to match the loudness of the third burst to the loudness of that portion of the burst pair that perceptually most resembles the third burst. This tendency was called a "principle of maximum similarity" (Zwislocki \& Ketkar, 1972). In agreement with it, listeners tend to match the

*The basic material of this article was presented at the 1972 spring meeting of the Acoustical Society of America (Sokolich \& Zwislocki, 1972). The work was supported in part by ONR Contract N00014-67-A-0378-0003 and in part by NIH Grant NS03950. loudness of the third burst to the total loudness when the time interval between the two bursts of a pair is so short that the bursts appear fused. At longer time intervals, when each burst is heard as a separate entity, listeners tend to match the loudness of the third burst to that of the second.

The experiments described in this article aim at an investigation of some intensity and frequency parameters of loudness enhancement and at further differentiation between loudness enhancement and summation. Preceding experiments conducted in our laboratory have shown that loudness enhancement of a tone burst following another sound burst is small when both bursts have the same loudness level. A more pronounced effect is found when the loudness of the first burst is increased. The latter finding was confirmed and extended by Galambos and his coworkers (1972) in a series of dichotic experiments. Our experiments overlap with theirs, but are monotic, differ in procedures, and have led to somewhat different results and conclusions.

\section{METHOD}

Our method was similar to that used previously (Irwin \& Zwislocki, 1971). The stimulus paradigm consisted of triads of monaural tone bursts presented repetitively to groups of 4-10 listeners with normal hearing. The listeners were requested to match the loudness of the third, comparison burst to that of the second, test burst, and to disregard the first, conditioning burst. The method of bracketing was strongly suggested to them. They controlled the intensity of the comparison burst by means of a smooth unmarked knob at tached to a logarithmic, continuou sly variable attenuator. Clues that could indicate to the listener the position of the attenuator setting were carefully avoided, and before each adjustment, the $E$ introduced randomized amounts of attenuation with the help of an auxiliary attenuator. The loudness matches were executed both in the presence and in the absence of the conditioning burst. The difference between the attenuator settings obtained under the two conditions was accepted as a measure of the change in loudness level of the test burst. To facilitate the loudness matches, the test and comparison bursts always had the same sound frequency of $1 \mathrm{kHz}$ and were of the same duration of $10 \mathrm{msec}$ at half-power 


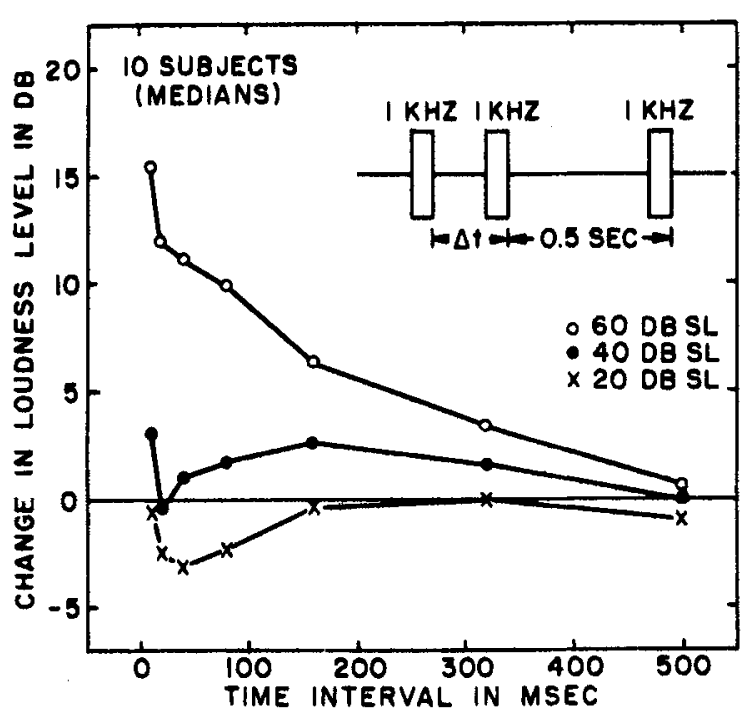

Fig. 1. Loudness-level changes of the second burst as a function of the interburst time interval, for three intensity levels of the first burst. The second burst, at $40 \mathrm{~dB}$ SL.

points. In order to confine their frequency spectra to one critical band, the bursts had approximately Gaussian envelopes. The envelope and duration of the conditioning burst was the same as of the other two bursts, except in one experiment, in which the burst duration was extended to $50 \mathrm{msec}$ without changing the onset and decay functions. In all the experiments, the test burst had a sensation level of $40 \mathrm{~dB}$. In the first three experimental serles, the loudness level of the test burst was determined as a function of the time interval between the conditioning and test bursts. In the fourth series, the sound frequency of the conditioning burst served as the independent variable. 1

\section{RESULTS}

In the first experimental series, the intensity of the conditioning burst was set consecutively at three levels: 20,0 , and $-20 \mathrm{~dB}$ relative to the level of the test burst. The median results obtained on 10 listeners are shown in Fig. 1. The effects of the conditioning sound intensity and of the interburst time interval are clearly apparent. They are in agreement with the results of preceding investigations. Little loudness enhancement is present when the conditioning and test burst are at the same loudness level (Irwin \& Zwislocki, 1971; Zwislocki \& Ketkar, 1972). A substantial loudness enhancement results when the intensity level of the conditioning burst is increased by $20 \mathrm{~dB}$ (Zwislocki \& Ketkar, 1972; Galambos et al, 1972). When the loudness level of the conditioning burst is decreased $20 \mathrm{~dB}$ below that of the test burst, it tends to pull the loudness of the test tone with it. Whatever the effect of the conditioning burst, it gradually decays to zero within about $500 \mathrm{msec}$.

Although the data of Fig. 1 basically agree with preceding results, their interpretation requires caution. As we mentioned in the introduction, listeners tend to judge the overall loudness of a burst pair rather than the loudness of the second burst at short interburst time intervals at which the bursts appear fused. Such a change of criterion may be the reason for the upturn of all the curves in Fig. 1 at the $10-\mathrm{msec}$ interval. A perceptual separation of the bursts appears particularly difficult when the first burst is considerably louder than the second. Great individual differences appear under these conditions, as shown in Fig. 2 for three listeners. Listener K.Z. seems to have changed her criterion almost suddenly between the intervals of 10 and $20 \mathrm{msec}$. Listener S.G. seems to have tried to separate the bursts even at an interval of $10 \mathrm{msec}$. Listener G.S. appears to have had the greatest difficulty with burst separation. The task becomes easier when the first burst has a different timbre from that of the second. Under such conditions, the loudness of the test burst tends to be depressed rather than enhanced at short time intervals (Zwislocki et al, 1974). On the basis of all this evidence, we must conclude that the curves of Fig. 1 may not reflect loudness enhancement at short time intervals but may be contaminated by loudness summation.

In a second experimental series, we explored the effect of the duration of the conditioning burst, which was extended to $50 \mathrm{msec}$. Its intensity level was again at 20,0 , or $-20 \mathrm{~dB}$ relative to the level of the test burst. Median results obtained on four listeners at the first two levels are shown in Fig. 3 (continuous curves). To avoid crowding, data gathered at the third level have been omitted. They do not contribute any additional insights. For comparison, the intermittent curves show corresponding results obtained on the same four listeners with the 10-msec conditioning burst. Although the data obtained with the 50 - and $10-\mathrm{msec}$ bursts are not identical, the differences between them are small and could be due to experimental error. In any event, increasing the duration of the conditioning burst by a

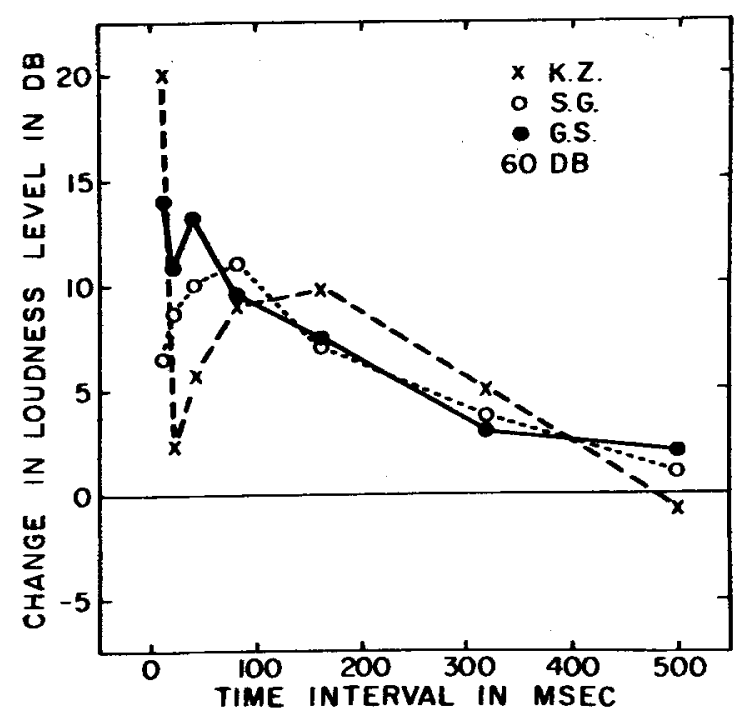

Fig. 2. Same as in Fig. 1, but for three individual listeners. Intensity level of the first burst, at $60 \mathrm{~dB}$, that of the second, at $40 \mathrm{~dB}$ SL. 
factor of 5 does not seem to have produced any overall increase in loudness enhancement. Further research is needed to ascertain whether slight changes in time pattern, which are evident in Fig. 3, are significant.

In a third experimental series, we made a first attempt at finding out whether the frequency relationship between the conditioning and test bursts had any effect on loudness enhancement. For this purpose, the sound frequency of the conditioning burst was changed from 1 to $4 \mathrm{kHz}$. A group of nine listeners participated, and the remaining experimental conditions were the same as in the first series. The results are plotted in Fig. 4. As can be seen, the effect of the conditioning burst disappeared almost completely, even at the highest intensity level of the burst. The upturn of the curves at the shortest time intervals is probably due to the spurious effect of loudness summation, as in the preceding experiments.

It should be noted that Scharf (1970) had found greater loudness summation for heterofrequency tone bursts than for homofrequency ones. In his experiments, the change in loudness level had remained small when the frequencies had been within one critical band, and had grown with the frequency difference at greater frequency separations. Since our results, shown in Figs. 1 and 4 , indicated an inverse frequency effect on loudness enhancement, we decided to investigate it in greater detail. For this purpose, the intensity level of the conditioning burst was fixed at $20 \mathrm{~dB}$ above the level of the test burst, and the interburst time interval was maintained at $80 \mathrm{msec}$. This interval was chosen to ensure that the conditioning and test bursts appeared as separate perceptual entities, and the effect of loudness summation was minimized. As can be seen in Fig. 2, loudness enhancement, when well separated from loudness summation, may be near its maximum at this interval.

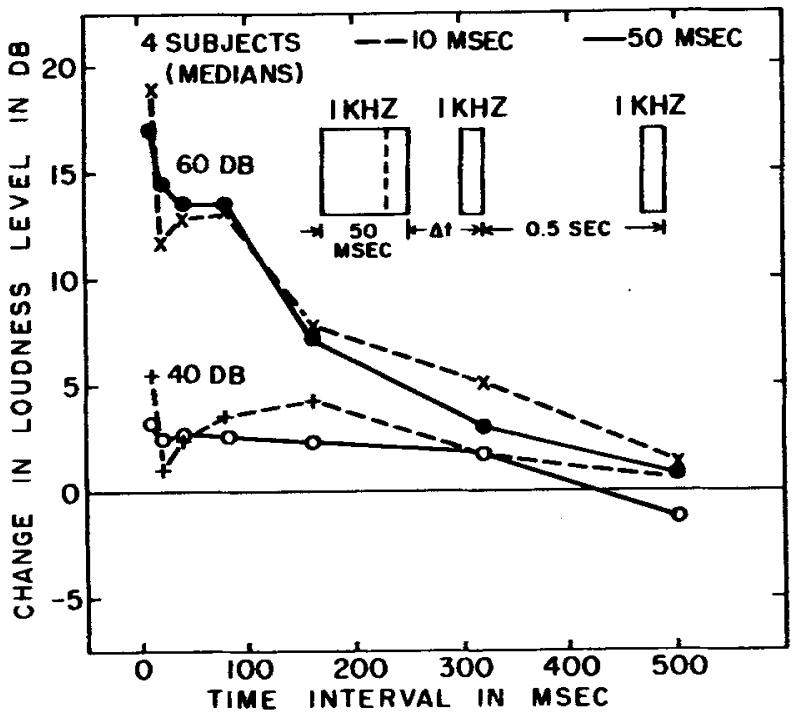

Fig. 3. Loudness-level change of the second burst as a function of the interburst time interval, for two durations and two intensity levels of the first burst. Second burst, at $40 \mathrm{~dB}$ SL.

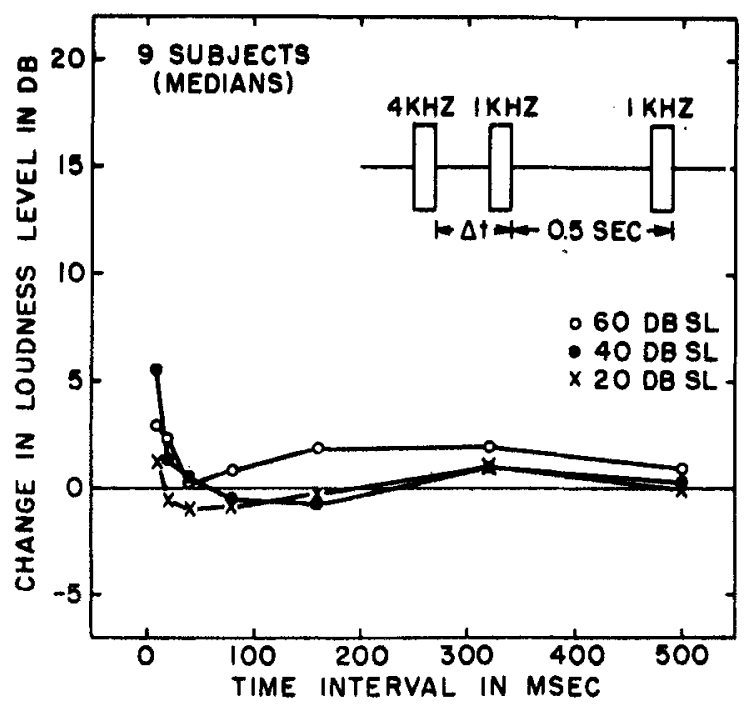

Fig. 4. Same as Fig. 1, but with the first burst at a much higher sound frequency than the second.

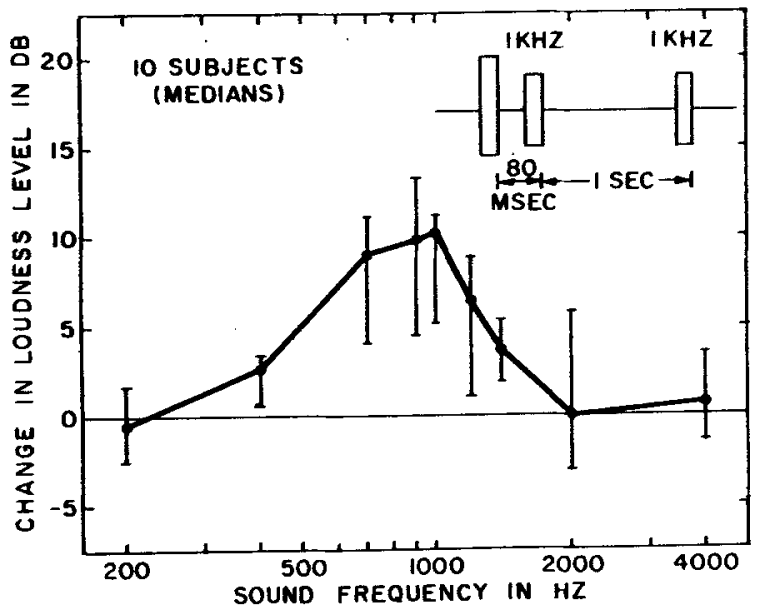

Fig. 5. Loudness-level change of the second burst as a function of the sound frequency of the first burst. Intensity level of the first burst, $20 \mathrm{~dB}$ above that of the second, which was at $40 \mathrm{~dB}$ SL. Interburst time interval, $80 \mathrm{~m} \mathrm{sec}$.

The results obtained on 10 listeners are shown in Fig. 5. The filled circles indicate the medians, and the vertical bars, the interquartile ranges of individual listeners. In spite of a rather large interlistener variability, a clear frequency pattern of loudness enhancement emerges. Maximum enhancement occurs when the frequency of the conditioning burst is near the frequency of the test burst. At lower frequencies of the conditioning burst, the effect decreases gradually to zero over about two octaves. At higher frequencies, the decay is more apid on $\log$ frequency scale, and disappears practically completely within one octave. The asymmetric pattern is consistent with the mechanical amplitude distribution in the cochlea (for instance, Békésy \& Rosenblith, 1951) and with the tuning curves of single units in the auditory nerve (for instance, Kiang, 


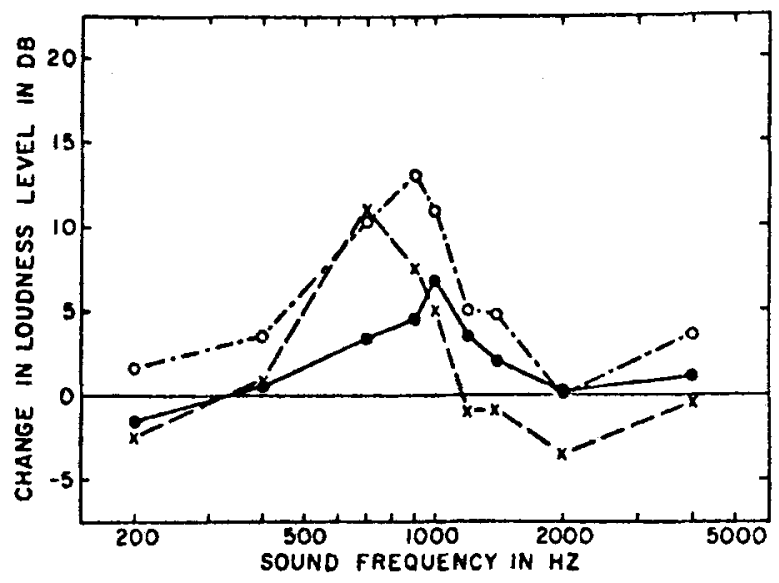

Fig. 6. Same as Fig. 5, but for three individual listeners.

1965); however, the maximum is much flatter than in the latter. The broad maximum in the median curve of Fig. 5 stems in part from the scatter of individual data. As Fig. 6 shows, the maximum loudness enhancement does not always coincide with the frequency of the test burst. In some listeners, it was shifted toward lower frequencies by as much as $300 \mathrm{~Hz}$, and the mean frequency of the maximum came out to lie $70 \mathrm{~Hz}$ below the frequency of the test burst. Nevertheless, the frequency bandwidths of individual curves, like those of Fig. 6, are larger than the critical bandwidth, which is on the order of $170 \mathrm{~Hz}$ at $1,000 \mathrm{~Hz}$ (Zwicker, Flottorp, \& Stevens, 1957).

\section{CONCLUSIONS}

Perhaps the most important outcome of our experiments is the demonstration that loudness enhancement has a fundamentally different frequency distribution than loudness summation. Whereas the former has its maximum when the conditioning and the test bursts are nearly at the same frequency, the latter has its minimum when the frequencies of the interacting bursts in a pair are within one critical band (Scharf, 1970). This finding gives strong support to the earlier conclusion that two distinct perceptual processes control loudness in pairs of consecutive sound bursts (Zwislocki \& Ketkar, 1972).

Loudness enhancement, which deals with the loudness of one burst rather than with the overall loudness of both bursts in a pair, appears to be more closely related to integrative processes at the threshold of audibility than is loudness summation. Like loudness enhancement, these processes are limited to reasonably narrow frequency bands called critical bands (Zwicker, Flottorp, \& Stevens, 1957) and have a time constant on the order of $200 \mathrm{msec}$ (Zwislocki, 1960). Loudness summation, on the other hand, occurs over wide frequency bands (Zwicker, Flottorp, \& Stevens, 1957) and long time spans (Zwislocki \& Ketkar, 1972).
Our data on loudness enhancement depart somewhat from those of Galambos et al (1972). They found a somewhat slower temporal decay of the phenomenon than we did. Moreover, they found that it depended on the duration of the conditioning burst and affected a wide range of frequency differences between the conditioning and test stimuli. Since their experiments were dichotic rather than monotic, it is possible that this difference is to blame for the discrepancies in the data. Nevertheless, it is also possible that their data have been affected by loudness summation. They used interburst time intervals of only $25 \mathrm{msec}$ at which perceptual separation of the bursts, required for loudness enhancement measurements, is very difficult. This being as it may, it should be clear that many more experiments are necessary to unambiguously define the characteristics of loudness enhancement.

\section{REFERENCES}

Békésy, G. v., \& Rosenblith, W. A. The mechanical properties of the ear. In S. S. Stevens (Ed.), Handbook of experimental psychology. New York: Wiley, 1951.

Cannon, M. W., \& Zwislocki, J. J. Loudness summation of pairs of short sounds. Journal of the Acoustical Society of America, $1970,48,71(\mathrm{~A})$.

Galambos, R. Bauer, J., Picton, T., Squires, K., \& Squires, N. Loudness enhancement following contralateral stimulation. Journal of the Acoustical Society of America, 1972, 52, 1127-1130.

Irwin, R. J., \& Zwislocki, J. J. Loudness effects in pairs of tone bursts. Perception \& Psychophysics, 1971, 10, 189-192.

Kiang. N. Y.-S. Discharge patterns of single fibers in the cat's auditory nerve. Researeh Monograph 35, M.I.T. Press, Cambridge, Mass., 1965.

Scharf, B. Dichotic summation of loudness. Journal of the Acoustical Society of America, 1969, 45, 1193-1205.

Scharf, B. Loudness and frequency selectivity at short duration. In R. Plomp and G. F. Smoorenburg (Eds.), Frequency analysis and periodicity detection in hearing. Leiden: Sijthoff, 1970 .

Sokolich, W. G., \& Zwislocki, J. J. On loudness enhancement of a tone burst by a preceding tone burst. Journal of the Acoustical Society of America, 1972, 52,141(A).

Zwicker, E., Flottorp, G., \& Stevens, S. S. Critical bandwidth in loudness summation. Journal of the Acoustical Society of America, 1957, 29, 548-557.

Zwislocki, J. J. Theory of temporal auditory summation. Journal of the Acoustical Society of America, 1960, 32, 1046-1060.

Zwislocki, J. J., \& Ketkar, I. Loudness enhancement and summation in pairs of short sound bursts. Journal of the Acoustical Society of America, 1972, 51, 140(A).

Zwislocki, J. J., Ketkar, I., Cannon, M. W., \& Nodar, R. H. Loudness enhancement and summation in pairs of short sound bursts. Perception \& Psychophysics, 1974, 16,91-95.

\section{NOTE}

1. In the first three experimental series, the listeners made three loudness matches per stimulus condition. The small number appeared acceptable because, as in preceding experiments of similar nature (Zwislocki et al, 1974), the intralistener variability was small, the total range of the three matches usually remaining under $4 \mathrm{~dB}$. The situation was different in the fourth experimental series, in which the frequency of the conditioning tone burst was varied. For some frequencies, the matches became unstable and their range increased to as much as $15 \mathrm{~dB}$ in some listeners. Accordingly, the experiments were replicated three times, increasing the number of matches per stimulus condition to 12. Means computed on the basis of the 12 matches had standard errors on the order of $2 \mathrm{~dB}-$ substantially, smaller than comparable measures of interlistener variability.

(Received for publication January 31, 1973; Revision received March 1, 1974.) 